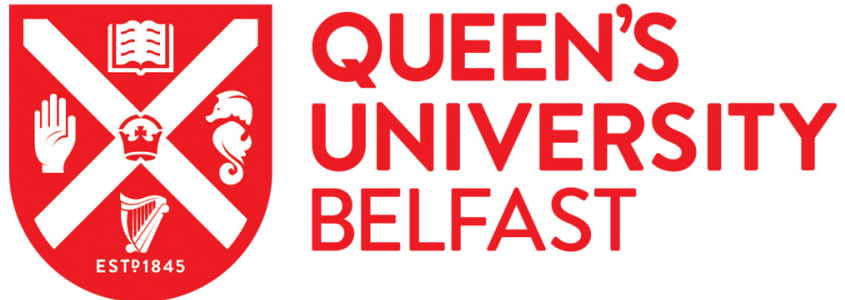

\section{A bibliometric/geographic assessment of 40 years of software engineering research (1969-2009)}

Garousi, V., \& Ruhe, G. (2013). A bibliometric/geographic assessment of 40 years of software engineering research (1969-2009). International Journal of Software Engineering and Knowledge Engineering, 23(9), 13431366. https://doi.org/10.1142/S0218194013500423

Published in:

International Journal of Software Engineering and Knowledge Engineering

Document Version:

Peer reviewed version

Queen's University Belfast - Research Portal:

Link to publication record in Queen's University Belfast Research Portal

Publisher rights

Copyright 2013 World Scientific Publishing. This work is made available online in accordance with the publisher's policies. Please refer to any applicable terms of use of the publisher.

\section{General rights}

Copyright for the publications made accessible via the Queen's University Belfast Research Portal is retained by the author(s) and / or other copyright owners and it is a condition of accessing these publications that users recognise and abide by the legal requirements associated with these rights.

Take down policy

The Research Portal is Queen's institutional repository that provides access to Queen's research output. Every effort has been made to ensure that content in the Research Portal does not infringe any person's rights, or applicable UK laws. If you discover content in the Research Portal that you believe breaches copyright or violates any law, please contact openaccess@qub.ac.uk. 


\title{
A BIBLIOMETRIC/GEOGRAPHIC ASSESSMENT OF 40 YEARS OF SOFTWARE ENGINEERING RESEARCH (1969-2009)
}

\author{
VAHID GAROUSI \\ Department of Electrical and Computer Engineering, University of Calgary, \\ Calgary, Alberta, T2N 1N4, Canada \\ Informatics Institute, Middle East Technical University \\ Ankara, Turkey \\ vgarousi@ucalgary.ca \\ GUENTHER RUHE \\ Department of Computer Science, University of Calgary, \\ Calgary, Alberta, T2N 1N4, Canada \\ ruhe@ucalgary.ca \\ Received (Day Month Year) \\ Revised (Day Month Year) \\ Accepted (Day Month Year)
}

Bibliometric rankings are quite common in the field of software engineering. For example, there are a series of ranking repeated every year which identify the top researchers and institutions in the international level in the field. There are also other studies to determine the most cited articles in software engineering journals, the most popular research topics in this area, or identify the top researchers and institutions in regional levels. However, there exists no existing bibliometric quantitative analysis of publications in the area of software engineering (SE), including relative and absolute growth in the number of all SE publications as well as an analysis among countries. This is the main goal and motivation of this article. Besides, this study intends to provide an overall quantitative trend of the software engineering papers, and compare that trend to research output in other areas of science.

The bibliometric study reported in this paper is motivated by the fact that understanding the amount of geographical research contributions to the field of software engineering can help identify different countries' level of commitment to support research activities in this area over years. We analyze how the contribution levels of top-ranked countries have changed over the years and how SE compares to other disciplines of engineering and science. Among the most interesting findings of this study are: (1) Over the 40 years, in total about $60 \%$ of the SE literature has been contributed by only $7 \%$ of all countries, (2) the SE research output of different countries does not necessarily correlate with their GDPs, (3) the share of contributions to the SE discipline by the American researchers has declined from $71.43 \%$ (in 1980) to $14.90 \%$ (in 2008), and (4) China is the country with the biggest share growth in the number of publications (from $0.82 \%$ of the entire SE publications in 1991 to $13.82 \%$ in 2009).

Keywords: Bibliometric study, software engineering, geographic bibliometric assessment.

\section{Introduction}


Software Engineering (SE) is an established discipline that has existed on its own for more than 40 years. The term software engineering first appeared in the 1968 NATO Software Engineering Conference, and was meant to provoke thought regarding the perceived "software crisis" at the time. With the increasingly ubiquitous application of the computing technologies, the maturity of SE has a considerable impact on almost every other discipline (i.e., it is hardly possible to imagine a modern society without heavy usage of software systems). Over the 40 years in consideration (1969-2009), the discipline of SE has gained a very substantial growth in depth and breadth of its research contributions. This paper is aimed at a quantitative analysis of this growth from an international and geographic perspective.

It is natural to raise various questions that are aimed to assess distinct facets of SE, for example: (1) Who are the active researchers in the field of software engineering?, (2) Which institutions (or research centers) are most the active in the field?, (3) Which research methodologies are used the most in this field?, (4) What are the active research topics in the field?, (5) Which countries are most the active in the field?, and (6) How the growth in the number of publications in Software Engineering compares to other disciplines of engineering and science?

There are various types of bibliometric studies in the field of Software Engineering (SE) addressing some of the above questions, e.g. [1-15]. For example, the on-going annual studies by Glass and colleagues [1-8] have been ranking the top researchers and institutions world-wide since 1996, addressing the questions (1) and (2) above. There are studies which rank the top SE researchers and institutions in regional levels (e.g., across Canada [10]).

There are also studies such as [12] and [13] to address questions (3) and (4) above, respectively. However, there exists no study to conduct a bibliometric study in the area to analyze the world's countries based on their number of contributions to the field, i.e., question (5) above. This is the main goal and contribution of the work reported in this article. This paper is motivated by the idea that understanding the geography of research contributions (measured by number of articles) to the field of SE can help identify different countries' level of commitment to support research activities, identify strengths, weaknesses, maturity levels, and opportunities in this area.

The authors of this paper were inspired to conduct this study after reading an excellent bibliometric study [16] in which 40 years (1969-2009) of publications in all scientific areas were studied. In particular, [16] analyzed the extent of each country's contributions to all areas of science. It was natural to ask similar questions in more focused areas of science, such as the discipline of SE. The study reported in this paper aims at addressing the following questions: Which countries have contributed the most to the field of SE since its inception (1969)? How have the top countries' contributions changed over years? How does the trend of SE publications volume compare to research output in other scientific areas?

To address the above questions, we mine statistical data from a well-known online database of research articles, the ISI Web of Knowledge [17], and provide results which 
can be of interest to both SE researchers and also research policy and decision makers. The remainder of this article is structured as follows. Goals and the research questions addressed by this paper are discussed in Section Error! Reference source not found.. Section Error! Reference source not found. discusses the related work. Section Error! Reference source not found. presents the research methodology used in this study. Section Error! Reference source not found. presents the results of our study. Finally, Section Error! Reference source not found. concludes the article and discusses directions of future work.

\section{Goal and Research Questions}

The goal of this study is to study the geographical volume of research contributions (i.e., number of papers) to the field of SE, for the purpose of analyzing the different levels of contributions of the countries and also identify trends during different years. Based on the above goal, we raise the following research questions (RQ's):

RQ 1. How is the overall trend of publications in the field of SE?

RQ 2. What are the top countries in terms of quantity of contributed papers to this field?

RQ 3. How do countries rank in terms of their SE research output relative to the following three metrics: (1) national GDP values, (2) research and development expenditure as a percentage of GDP, and (3) population sizes?

RQ 4. How have the contributions of the top countries changed over years?

RQ 5. What are the levels of contributions to the field in different regions, i.e., intercontinental comparisons, emerging economies, EU countries, and the Middle Eastern countries?

RQ 6. How does the trend of SE publications compare to research output in all other areas of engineering and science?

As the time range to do this bibliometric study, we searched for the earliest paper in SE in the following search engines: IEEEXplore, ACM digital library, Google Scholar and ISI Web of Knowledge. The earliest paper we found in the archive was the classical paper [18] published 1969 by Bernard A. Galler, being the ACM president at that time, entitled "NATO and Software Engineering?", which was published in the Communications of the ACM. Rather than being a technical paper, it was a letter-type article where the author provided an interesting report about the NATO's first conference on SE. We thus set 1969 as the start date of the period under study.

For the end date, we wanted to set a year to ensure that all the articles from that year will be included in the search engine we were using. We also looked at other bibliometric studies [1-15] to see what other authors are choosing in this regard. We found that we should give at least a two-year slack time before our study's execution time (early 2011). This resulted in having 2009 as the end date of the range under study. Thus, we analyzed the 40-year window: from 1969 to 2009. 


\section{Related Work}

Bibliometric rankings are quite common in field of SE [1-15]. Rankings have been proposed based on both sole counting of papers and also citation counting.

Perhaps, the most well-known ranking in this area are the on-going annual survey by Glass and colleagues [1-8] which have so far ranked the top researchers and institutions world-wide since 1996 until 2008. The annual ranking done by Glass and colleagues [1$8]$ is based on paper counting without considering number of citations. Ranking is based on the weighted scores of the number of papers published in six selected journals and one magazine which have the highest impact factors among all software engineering journals, and are widely perceived as the top journals in the area. They are: (1) IEEE Transactions on Software Engineering (TSE), (2) ACM Transactions on Software Engineering and Methodology (TOSEM), (3) Elsevier Journal of Systems and Software (JSS), (4) Wiley Journal of Software Practice and Experience (SPE), (5) Springer Journal of Empirical Software Engineering (EMSE), (6) Elsevier Journal of Information and Software Technology (IST), and (7) IEEE Software Magazine.

Some critics of the evaluation method used by Glass and his colleagues believe that correctness, importance, novelty, and overall contribution of each paper should be given greater consideration than the number of publications [19]. However, most researchers agree that an assessment on these grounds will certainly be influenced by subjective factors such as the competence or bias of the reviewer [20]. In addition, the time investment required to adequately review each paper significantly limits the number of publications that can be included in a survey. Citation counting has been proposed as an enhancement to publication counting, although Parnas [19] observed that a citation might well imply a negative critique or simply a neutral reference as part of a general summary of related work. While the development of a more comprehensive and accurate metric for the assessment of researchers and institutions is a worthwhile goal, the rankings provided by publication counting can still be useful [21].

Ren and Taylor followed an impact-factor-based approach and presented a ranking in 2007 [9] which provided different results (not that different though) compared to the assessments done by Glass and colleagues [1-8]. As part of their study, Ren and Taylor also developed a Java tool (which is freely available online [22]) which can be used by other researchers. To weigh the papers and produce the ranking, their tool supports two types of bibliometric metrics: impact factors, and h-index.

We recently used the Java tool developed by Ren and Taylor [22] to conduct a Canadawide ranking of SE scholars and institutions [10], based on both impact factor, and hindex values. A break-down by different provinces of Canada was also done. Furthermore, we took a further step and conducted an initial inter-provincial research efficiency analysis, by taking as input the total dollar value of research funds received by each province, and relating it to the number of generated papers as the output.

Published in the Journal of Scientometrics [11], McCain et al. used bibliometric and knowledge elicitation techniques to map a knowledge domain for SE in 1990s. Mappings of the intellectual and cognitive structure of SE were conducted using three techniques: 
(1) author co-citation analysis, (2) pathfinder network analysis, and (3) card sorting, as a knowledge elicitation method. Co-citation counts for 60 prominent SE authors over the period of 1990-1997 were gathered from a scholarly database called SciSearch. The study revealed interesting findings including a co-citation and a pathfinder network map for the SE domain in the 1990's.

Glass et al. conducted an analysis of the research and research methods in the SE literature as of 2002 [12]. They examined 369 papers in six leading research journals in the SE field. From that examination, the authors concludes that SE research is diverse regarding topic, narrow regarding research approach and method, inwardly-focused regarding reference discipline, and technically focused (as opposed to behaviorally focused) regarding level of analysis. The authors however passed no judgment on the SE field as a result of these findings. Instead, they presented them as groundwork for future SE research efforts.

The study by Caia and Card [13] analyzed the active research topics in SE. The authors considered 7 top journals and 7 top international conferences in SE and examined all the 691 papers published in these journals or presented at these conferences in year 2006. The paper [13] reported several interesting findings: (1) 73\% of journal papers focus on $20 \%$ of subject indexes in SE, including testing and debugging, management, and software/program verification, (2) $89 \%$ percent of conference papers focus on $20 \%$ of subject indexes in software engineering, including software/program verification, testing and debugging, and design tools and techniques, and (3) the average number of references cited by a journal paper is about 33 , whereas this number becomes around 24 for a conference paper.

Furthermore, Wohlin conducted two consecutive studies in 2000 and $2001[14,15]$ to determine the most cited articles in the SE journals. The objective of the analysis was to identify and list the articles that have influenced others the most as measured by citation count. An understanding of which research is viewed by the research community as most valuable to build upon may provide valuable insights into what research to focus on now and in the future. Based on the analysis, a list of the 20 most cited articles was presented here. The intention of the analysis was twofold. Afterwards, the authors of the most cited articles in 2001 were invited to contribute to a special section of the Elsevier journal on Information and Software Technology (IST).

We were able to find only two articles $[23,24]$ which, as part of their studies, present a bibliometric assessment of contributions in terms of their origination. These two studies are systematic literature reviews (SLR) and both conducted by Harman and his team at University College London, UK. However, these two studies are on focused sub-areas of SE, namely software mutation testing [23], and search-based SE [24]. Both studies measure the number of publications by each nation in these two areas and provide interesting trends and insights.

To put all the above related works in summary, there exists no study to conduct bibliometric analysis among the world's countries based on their number of contributions to the field of SE. This is the goal and contribution of the work reported in this article. 


\section{Research Methodology}

Details on our data source and also our methodology to search for data and statistics are presented in Sections 4.1 and 4.2, respectively. Potential threats to the validity of our study and the steps we have taken to minimize them are discussed in Section 4.3.

\subsection{Data Source}

As our source of information, we evaluated IEEEXplore, ACM Digital Library, ISI Web of Knowledge (WoK), Scopus and Google Scholar. To choose the best data source, we reviewed several studies conducted for the sole purpose of identifying most comprehensive data sources for bibliometric studies, e.g., $[25,26]$. Databases such as IEEEXplore, ACM Digital Library and Google Scholar seemed to have a limited search pool in their databases. For example, IEEEXplore only provides the papers published by the IEEE. Similarly, the ACM Digital Library only has the papers published by the ACM. The authors of [26] have done an interesting analysis by calculating the correlation of the number of papers by country, based on inputs from Web of Knowledge (WoK) and Scopus. Interestingly, the correlation is very high $\left(\mathrm{R}^{2}=0.994\right)$. Thus, we almost had two equal alternatives to choose from. If we were to conduct our study with either choice, we would expect to get similar results. Between the two similar alternatives, we selected WoK as it was used by similar studies already.

ISI WoK [17] is an online academic database provided by Thomson Scientific's Institute for Scientific Information. As of April 2011, when this study was conducted, the database had 95,515,221 papers from almost all areas of science in its archive. This database has also been used by various bibliographic studies in different areas (e.g., $[16,27])$ in the past. WoK covers publications in 256 different disciplines in science, engineering, arts, and humanities, [17]. Its index includes more than 10,000 of the highest impact journals worldwide, including Open Access journals and over 110,000 conference proceedings. WoK contains, in its index, all the major SE journals (including those published by the IEEE, ACM, Springer, Wiley and Elsevier). Also the proceedings of top conferences in the area are also included.

\subsection{Search Methodology}

To search for the SE papers published by researchers of each country as indexed by WoK, we entered the "software engineering" phrase in the Topic field of the WoK search page, and the name of the country in the Address field. In the case of UK, it is sometimes written in full as "United Kingdom" and relates to/includes the geographical locations of England, Scotland, Wales, and Northern Ireland. We conducted searches using each of the above six terms and subsequently added up the results. 
To ensure accuracy of the paper pool, we used a filtering approach to make sure that only papers with the "software engineering" phrase appearing in the relevant subject areas are included in our pool. To do this, WoK has a filtering mechanism based on pre-defined subject areas. In the entire list of subject areas, there was no "software engineering" term. The two closest ones were "computer science" and "engineering". We filtered papers using these two areas, as shown in Fig. 1-(a). This filtering reduced the initial pool of 34,278 papers to 26,624 papers. Filtering using the above subject areas was done to exclude irrelevant papers, e.g., our initial result pool could include a paper in the domain of chemistry which might have mentioned a sentence such as: "we used the software named X to run our simulations".

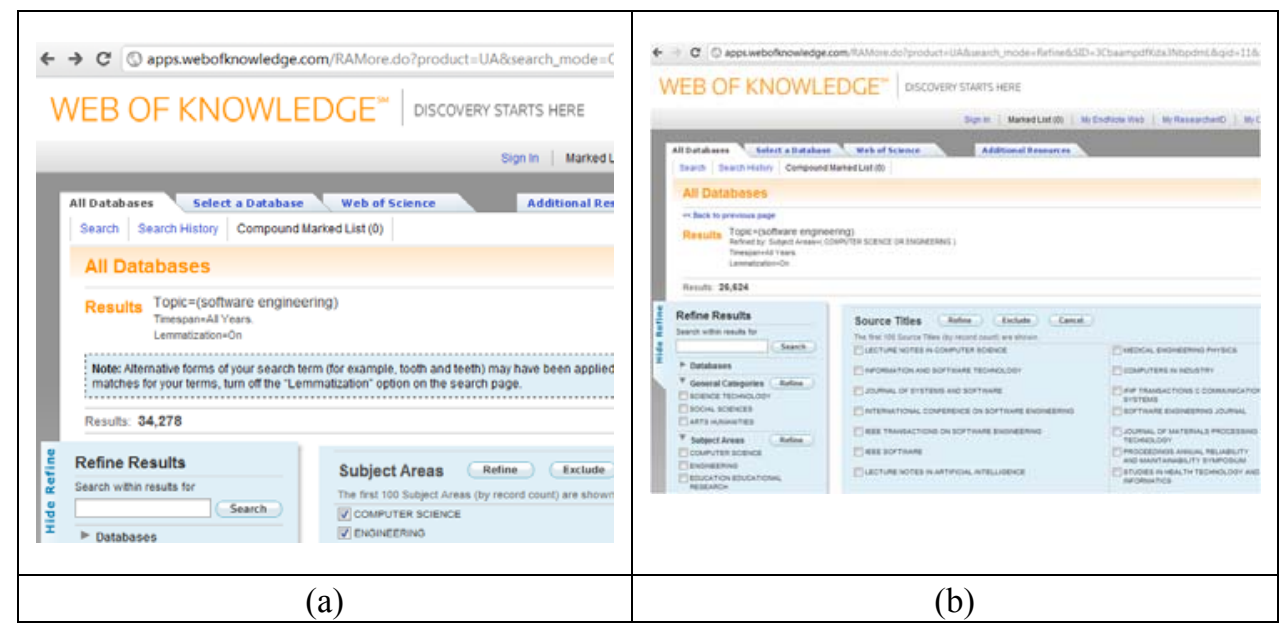

Fig. 1. Searching (a) and filtering (b) of the data in WoK to ensure accuracy of results.

As a second step, to ensure accuracy of the paper pool, we verified the venues of the papers. In the WoK's search page, venues are referred to as "Source Titles", as shown in Fig. 1-(b). We checked to see if the pool contains all main SE venues (journals and conference proceedings). The list of SE journals that we specifically checked for were taken from the series of studies done by Glass et al. [1-8]: (1) IEEE Transactions on Software Engineering (TSE), (2) Journal of Systems and Software (JSS), (3) ACM Transactions on Software Engineering and Methodology (TOSEM), (4) Information and Software Technology (IST), (5) IEEE Software, and (6) Software: Practice and Experience (SPE).

We checked the inclusion of the following major SE conferences [28]: International Conference on Software Engineering (ICSE), International Symposium on the Foundations of Software Engineering (FSE), International Conference on Automated Software Engineering (ASE) and International Conference on Software Maintenance (ICSM). To further validate the accuracy of the papers in our pool, we used the two wellknown accuracy metrics from the information retrieval and data-mining literature: 
precision and recall [29]. Precision is the fraction of retrieved instances that are relevant, while recall is the fraction of relevant instances that were retrieved.

To calculate the precision metric, the approach is to verify that each of the 26,624 papers in the pool is a relevant SE paper. It would have been infeasible to do this task manually for all the papers. To keep our workload manageable, we randomly verified a manageable number of 200 papers in the pool. Only two papers in the randomly chosen subset of the pool did not appear to have a core SE focus, but they were in other engineering fields and using software systems for engineering purposes. The precision rate thus was $99 \%$.

To measure recall, we selected a representative paper set outside the pool. For this purpose, we chose the ICSE best papers list".We then verified whether the generated pool of papers included those papers. All the papers were in the pool, denoting that the recall rate was $100 \%$. With the precision rate of $99 \%$ and recall rate of $100 \%$, we concluded that the accuracy of our data set and paper pool is sufficiently high.

\subsection{Threats to Validity}

For this study, the following threats to validity are applicable: internal, construct, and external validity. Internal validity refers to the certainty that the different research questions studied are properly based on the papers selected. To search for the SE papers published by researchers of each country, we entered the "software engineering" phrase in the Topic field of the WoK search page. However, some papers might contain only the term SE and not the full name. Thus, there is a potential threat that those papers were not retrieved.

We addressed internal validity and reduced (selection) bias in two ways. Firstly, we chose the ISI Web of Knowledge (WoK) which was pre-evaluated to be the most comprehensive data source. Secondly, to validate the accuracy of the paper selection, we used the two well-known accuracy metrics from the information retrieval and datamining literature: precision and recall [29] (Section 4.2). Due to limited time resources in manual application of the metrics precision and recall, they were applied on a very small set (200 papers) only.

Construct validity refers to the relationship between theory and observation. Does the proposed measurement actually represent the topic of investigation? In our context, our goal was to conduct a bibliometric assessment of countries. We used the simplest bibliometric metrics, i.e., number of papers, for this approach. We would have liked to use more sophisticated metrics such as h-index [30] and impact factor [31]. However, we did not have the data for these metrics, and thus we could not use those metrics. We plan to conduct ranking studies using such advanced metric in future works. In addition, for RQ 3, GDP, R\&D expenditure and population size was taken into account to relate number of papers to the values of the respective attributes. All these measures are commonly accepted and frequently used in similar contexts to characterized financial and size dimensions of the different countries.

*http://www.icse-conferences.org/mostinfluential.html 
External validity refers to the generalizability of results. All the steps performed have been made transparent. All the papers were taken from WoK are publicly available. Since our pool had high level of precision, we believe that it is possible to replicate and also generalize our results. One threat to external validity is that we only covered data up to 2009.

\section{Results}

The results of the study are reported in this section. The results are structured based on the six research questions raised in Section Error! Reference source not found.. They are presented in Sections Error! Reference source not found. to 5.6, respectively.

\subsection{RQ 1: Overall Trend of Publications}

As to the total number of SE papers in this database, as of early April 2011 when the data collection for this study was made, 26,624 papers were found.

The histogram of the number of SE papers per year in the WoK from 1969-2009 is shown in Fig. 2. For better rendering, the y-axis is on logarithmic scale. There are 10 papers or less per year until 1979. Thus, to have a reasonable data pool for our study, we use 1980 as the starting year for most of the analyses in the rest of Section 5.

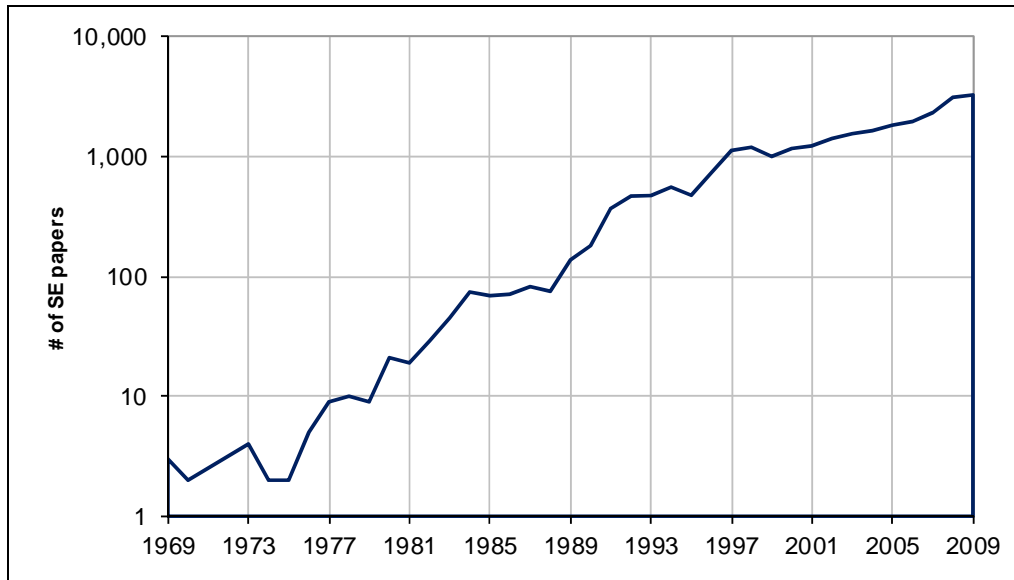

Fig. 2. Histogram of the number of SE papers per year in the WoK. The y-axis is in logarithmic scale.

It becomes clear that the number of papers per year has grown very rapidly over the last 40 years. To further illustrate this point, we also quantitatively analyzed the growth rate of the number of SE papers from each year to the next year, starting from 1981. Results are shown in Fig. 3, e.g., the number of SE papers as indexed by WoK in 2007 and 2008 were 2,311 and 3,101, respectively, yielding in a relative growth rate of $34.2 \%$. We can see from Fig. 3 that the overall growth rate is mostly positive, only having negative rates in five years in this time window. The highest annual growth rate was in year 1991. 


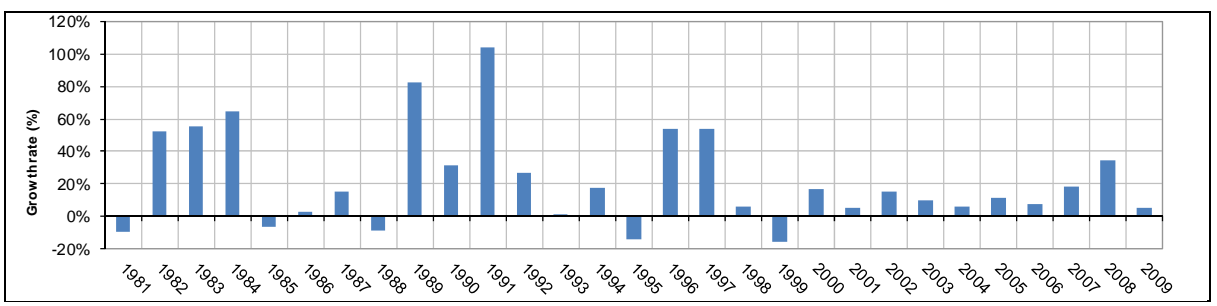

Fig. 3. Annual growth rate of the number of SE papers for the period of 1981 to 2009 .

\subsection{RQ 2: Ranking in Terms of Quantity of Papers}

The breakdown of all the SE papers classified based upon the authors' origin is shown in Fig. 4. If a paper had several authors from different countries, the breakdown counts the paper for each of the involved countries (this is the way the WoK handles the search results). In case of two affiliations of the same author, both of them were taken.

For brevity, the top-15 countries (based on the number of SE papers) over the period of 40 years are shown in Fig. 4 . The US is the clear leader, followed by UK and China. The remaining 178 (=193-15) countries, not shown in this list, have contributed altogether by about 8,400 papers in total (or just about $31.4 \%$ ). This volume of contribution is slightly less than those of the three top countries combined. This also means that, at this point in time, $68.6 \%$ of the SE literature has been contributed by the top 15 countries.

Most of the top 15 countries are from the western world, while only three countries from Asia are also present in the top 15 (China, Japan, and South Korea). There seems to be a good mix of countries from different parts of the world in this list. We can observe low contributions levels from Africa and South America (except Brazil).

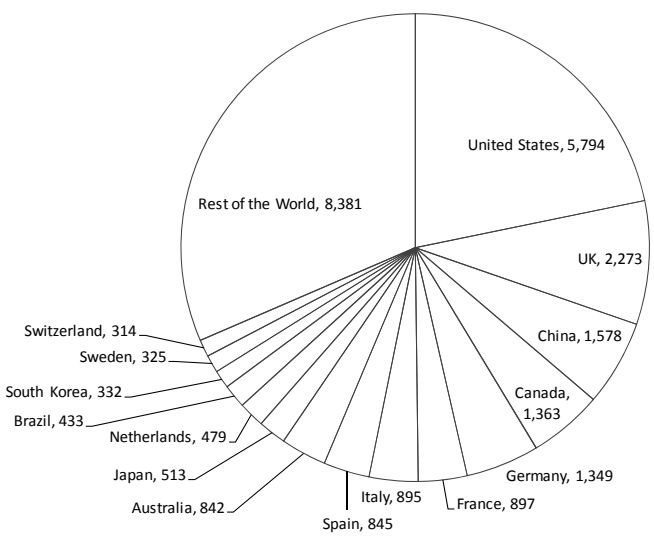

Fig. 4. Top 15 countries. 


\subsection{RQ 3: Ranking of Countries based on GDP, R\&D Expenditure and Population Size}

It would also be interesting to compare the SE output of each country with three other related metrics: (1) national GDP values, (2) research and development expenditure as a percentage of GDP, and (3) population sizes. These comparisons are presented next.

\subsubsection{National GDP Values}

In order to better relate the number of publications to the strength of each country's economy, as a follow-up analysis, we considered the GDP values while analyzing the SE research output of each country. We extracted the 2009 GDP values of different countries from the data provided by the World Bank [32].

Since the GDP amounts are reported for each year, we decided to use the last year (2009) of our date range as the reference for comparisons. The scatter plot in Fig. 5 visualizes the top tanking countries with respect to their number of SE papers in 2009 versus their 2009 GDP values. To facilitate the analysis, the regression trend-line is also shown. For brevity, the top 15 countries ranked by their number of SE papers are shown in this chart. A regression line is also shown. GDP is an established measure for the volume/size of a country's economy. Even though GDP of a country is not the most precise measure for financial support of SE research in a country, we have used the GDP in this section to relate the number of SE papers to GDP. In the following section, we will correlate the quantity of SE publications versus another relevant metric, i.e., the Research and Development (R\&D) expenditure of each country as a percentage of its GDP.

As it can be seen in Fig. 5, most of the countries lies in the bottom-left corner of the chart, denoting that their GDP and SE research output is in smaller scale compared to GDP leading countries such as US, China, Japan and Germany. Interesting observations can be made using this visualization:

- In both charts, the US is the obvious leader in both the GDP and SE research output.

- Countries above the regression trend-line have an above-average portion of publications relative to GDP, e.g., US, Canada, China, Spain, and UK.

- Countries under the regression trend-line denote those which are relatively higher in GDP, but the SE research output in those countries is not proportionally high, e.g., Japan, Germany, France and Italy.

- Although the 15 points in Fig. 5 do not seem to be that close to a linear relationship, but once calculated, the correlation between the GDP values and the number of papers in 2009 for these 15 countries is quite high $\left(R^{2}=0.91\right)$. 


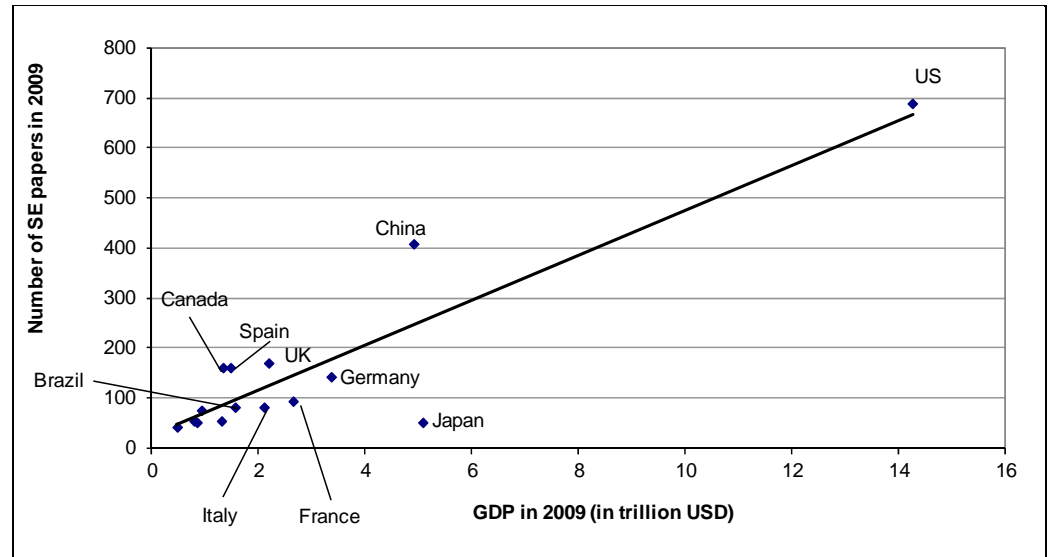

Fig. 5. Top 15 countries with respect to their SE research output in 2009 papers versus their 2009 GDP values.

Another way of considering GDP values is to normalize the above absolute number of papers by countries' GDP values. For this purpose, and measuring GDP in trillion USD, we define the following metric called GPD-Normalized paper output per year $y$ and country:

The above metric expresses the relative dollar cost (from the countries' GDP) for each SE paper. The bigger the value, the more efficient that country becomes in publishing SE papers. There have been similar measurements and studies in other disciplines using this approach, which is often referred to estimation of "cost of research", e.g., in health sciences [33], grants funded by the American National Science Foundation (NSF) [34], and research projects funded in the universities of the Canadian province of Quebec [35]. Note that those studies [33-35] were able to calculate or estimate the dollar amount of research support for the corresponding discipline(s). However, we were not able to find publicly-available sources for the SE research in each country. As discussed above, we use the GDP values in this study as an approximation for that purpose.

Since the GDP amounts are reported for each year, we decided to use the last year of our date range as the baseline year for comparisons. We ranked the countries by the number of their 2009 SE papers and extracted the top 30 countries. We then calculated the above metrics for each country. The ranking of the top 30 countries according to the above metric in year 2009 is shown in Fig. 6. We have also shown the absolute number of 2009 papers published by each country in this figure. In terms of the above metric, Ireland ranks first. With a GDP of about 230,000 billion USD in 2009 [32], SE researchers from Ireland are the (co-) authors of $31 \mathrm{SE}$ papers in 2009, as per the WoK database. 


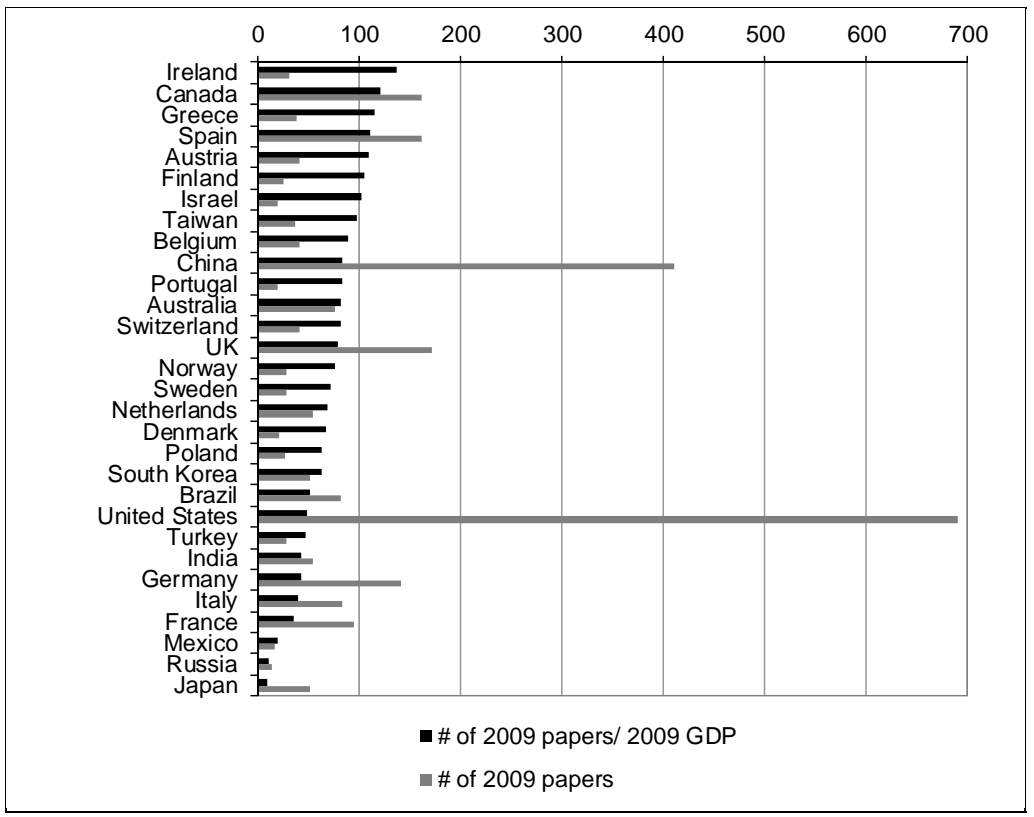

Fig. 6. Top 30 countries ranked according to GDP-Normalized Paper Output in 2009.

\subsubsection{Research and Development ( $R \& D$ ) Expenditure (\% of GDP)}

Another relevant metric to correlate the quantity of SE publications with is the Research and Development (R\&D) expenditure of each countries as a percentage of its GDP. We extracted the 2009 GDP values of Research and Development (R\&D) expenditure (\% of GDP) from the World Bank database [36].

Fig. 7 visualizes, as a scatter plot, the top 15 countries with respect to their total SE research output versus their R\&D expenditure as a percentage of their GDP. Again, the US clearly stands out. Among the top 15 countries, South Korea has the highest relative R\&D expenditure (3.36\% of its GDP). With a relatively small R\&D expenditure (only $0.16 \%$ of its GDP), China is ranking $2^{\text {nd }}$ in SE research output (i.e., by being the second top-most on the Y-axis). Countries such as China and Germany are above the regression line, denoting their above-average performance in SE research output in terms of their R\&D expenditure ratio, and several countries, as listed in Fig. 7, are below the regression line. 


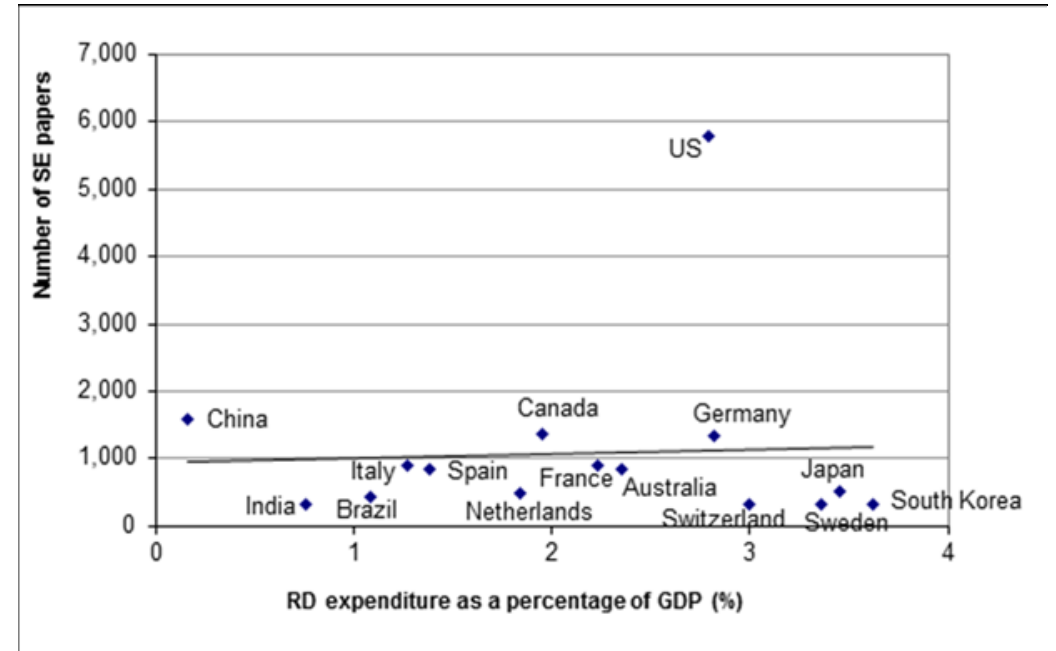

Fig. 7. Top 15 countries with respect to their total \# of papers versus their R\&D expenditure as a percentage of GDP (\%)

\subsubsection{Population Size}

Considering the population of each country, one may wonder how the population of each country correlates to its SE publication output. Fig. 8 visualizes, as a scatter plot, the top 15 countries with respect to their total SE research output versus their latest (2012) population sizes. The x-axis (population) is on a logarithmic scale. Again, the US clearly stands out. India with a huge population has a relatively small SE research output, compared to the other countries. Switzerland with a population of 1.2 million, which is only about $0.1 \%$ of India's population, ranks higher than India in SE research output.

Switzerland, Germany and Canada are above the regression line, denoting their aboveaverage performance in SE research output in terms of their population sizes. The following countries are below the regression line: China, France, Italy, Spain, Japan, Netherlands, Brazil, South Korea, Sweden and India. Australia is on the regression line. 


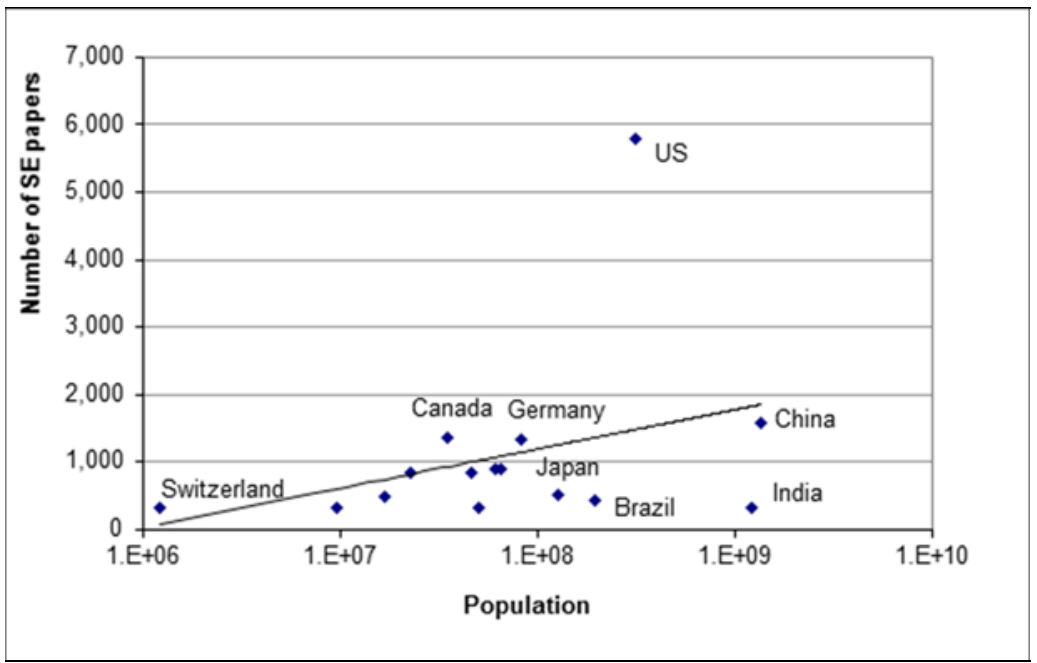

Fig. 8. Top 15 countries with respect to their total \# of SE papers versus their latest (2012) population sizes.

\subsection{RQ 4: Trend of Contributions by Country}

To get an in-depth understanding of how the top countries have contributed relatively to the area over years, we analyzed at the yearly contributions of researchers from those countries. To keep the visualization readable, Fig. 9 shows the trend data for the period 1980-2009 for the top-5 countries (US, UK, China, Canada, and Germany) in comparison with the rest of the world.

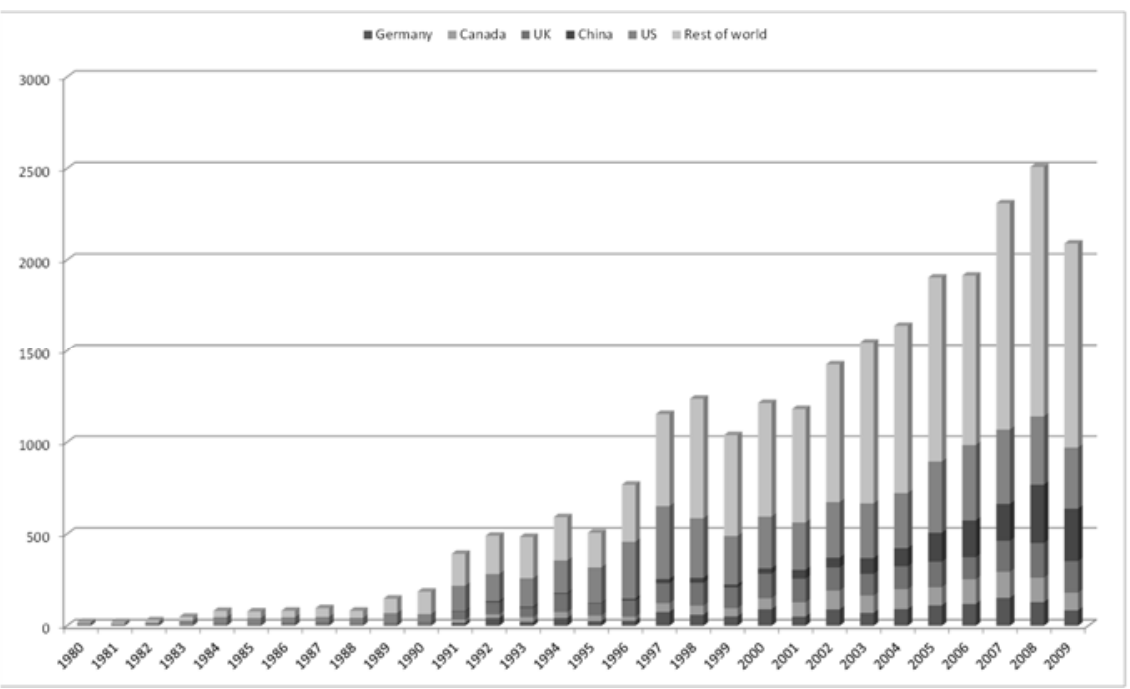

Fig. 9. Top 5 countries’ SE research output from 1980-2009: absolute values. 
The US researchers have taken the lead in all of the years. However, as years have passed, the US share of contributions has slightly declined. The maximum US contribution (in relative measures) was in 1980 (71.43\%) and the minimum was in 2008 $(14.90 \%)$.

There is no single paper in the database from Chinese researchers before 1990. However, especially after 1995, an accelerated increase in number of research papers by Chinese researchers seems to occur. Their share has increased from $0 \%$ in 1990 and before, to $0.82 \%$ in 1991 to $13.82 \%$ in 2009 . The contribution shares of Canada, Germany, and the UK in the years after 1990 are only increasing slowly. This seems to indicate that the researchers from those countries are constantly keeping up their publications volume similar to the world average.

\subsection{RQ 5: Regional Contribution}

It would be interesting to compare the trend of publications based on regional classifications. To keep our efforts manageable, we compare the volume of contributions according to the following classifications: continents, countries of the European Union, countries of the so-called emerging economies, and the Middle Eastern countries.

\subsubsection{Continents}

The SE research outputs of the world's six continents are depicted in Fig. 10. North and South America has been shown as two separate continents. It is interesting to observe that Europe's SE research output has surpassed that of North America after the middle years of the range under study. During the last ten year, Asia has made the biggest increase over a period of the last ten years. With the exception of 2009, SE research from South America has seen a sharp increase in latest years. 


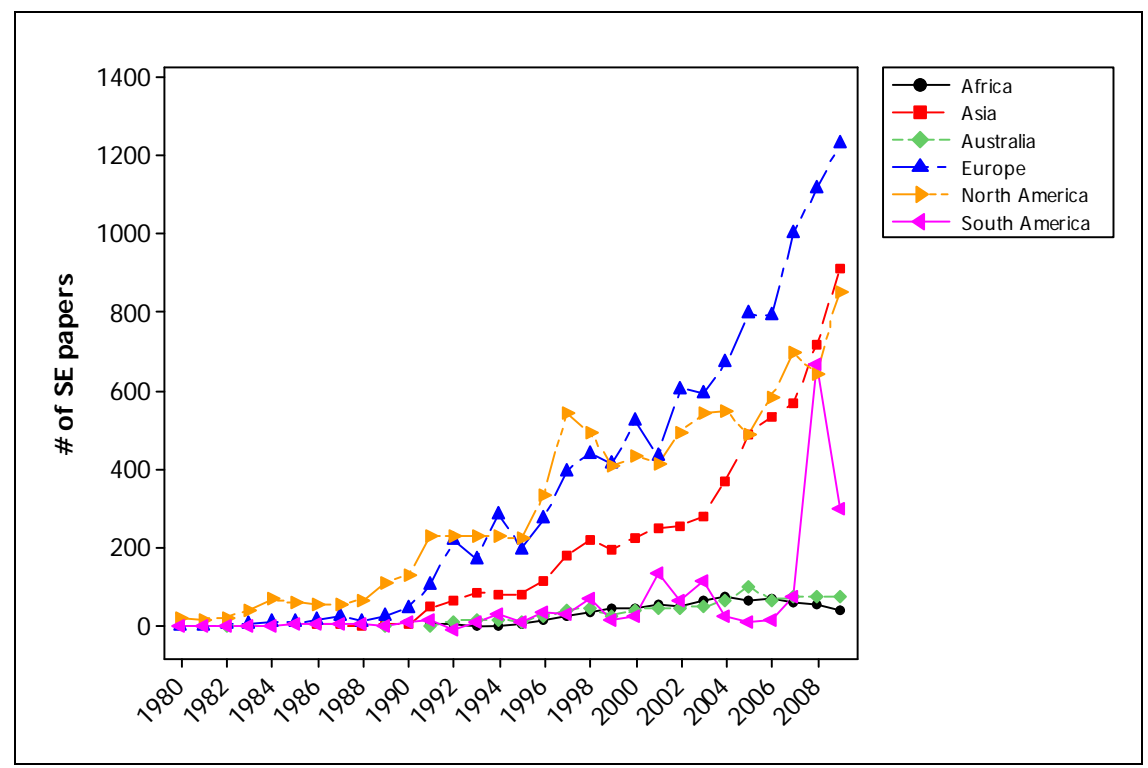

Fig. 10. SE research output by continents.

\subsubsection{European Countries}

Europe has always been a major hub for SE research and practice. The first SE conference was held in Germany by the NATO in 1968. SE research outputs of the top20 European countries in terms of their 2011 GDP are depicted in Fig. 11-13. To allow a meaningful comparison, the top 20 countries were subdivided into three groups:

- Group 1: Germany, France, UK, Italy, Russia and Spain (2011 GDP above 1000 Billions \$US).

- Group 2: Netherlands, Turkey, Switzerland, Sweden, Poland, Belgium (2011 GDP above 500 Billions \$US).

- Group 3: Norway, Austria, Denmark, Greece, Finland, Portugal, Ireland, Czech Republic (2011 GDP above 200 Billions \$US). 


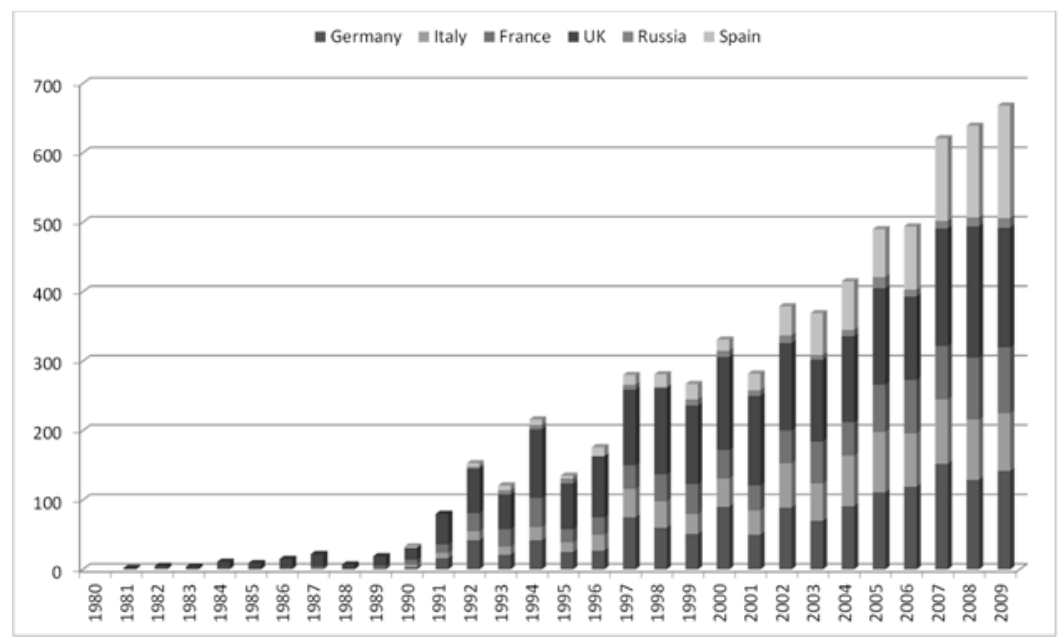

Fig. 11. Evolution of SE research output for European Group 1 countries.

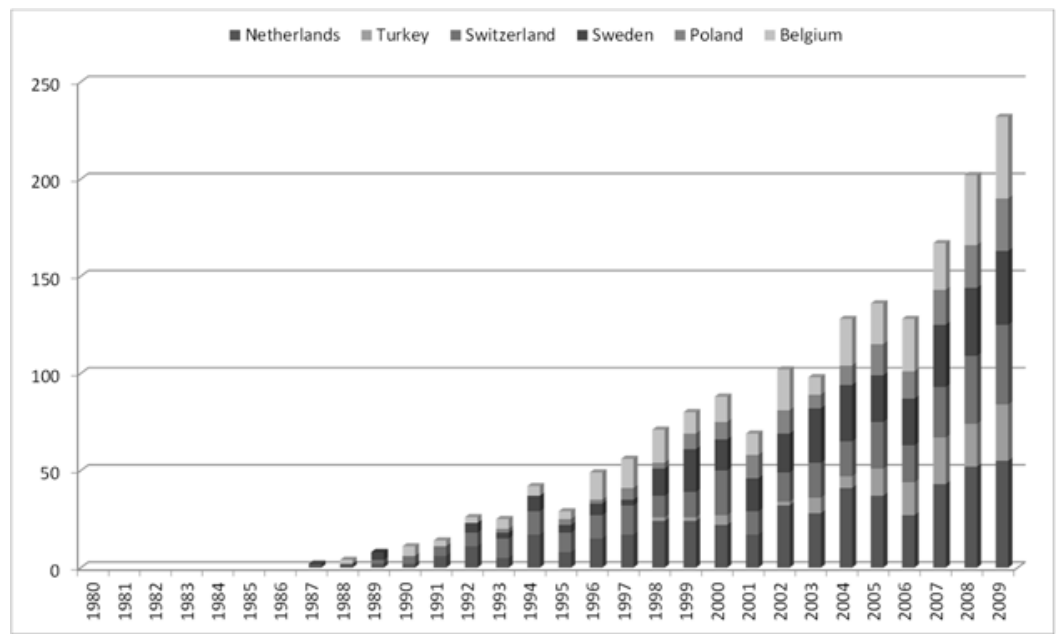

Fig. 12. Evolution of SE research output for European Group 2 countries. 


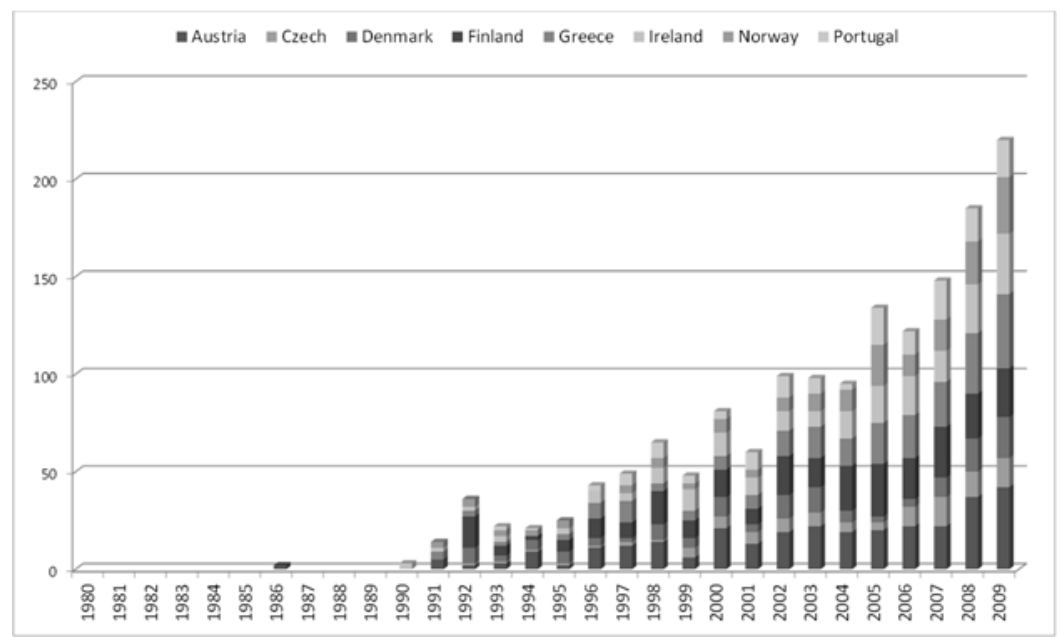

Fig. 13. Evolution of SE research output for European Group 3 countries.

The UK maintained a leading position over all the years. Netherlands and Austria is the leader among the Group 2 resp. Group 3 countries. Among all countries, Spain has made most relative progress over the last five years.

\subsubsection{Emerging Economies}

There are various metrics and discussions in the international business community to identify the emerging countries in the world. Perhaps, the most common list of the emerging countries used by many sources (e.g., [37]) is the so-called BRIC (Brazil, Russia, India, and China), also known as the "Big Four" [38]. The data for these four countries are shown in Fig. 14. It is evident that the SE research output from China has seen a tremendous increase since early 2000. The other three countries (Brazil, Russia and India) do not have a major increase in their SE research output. 


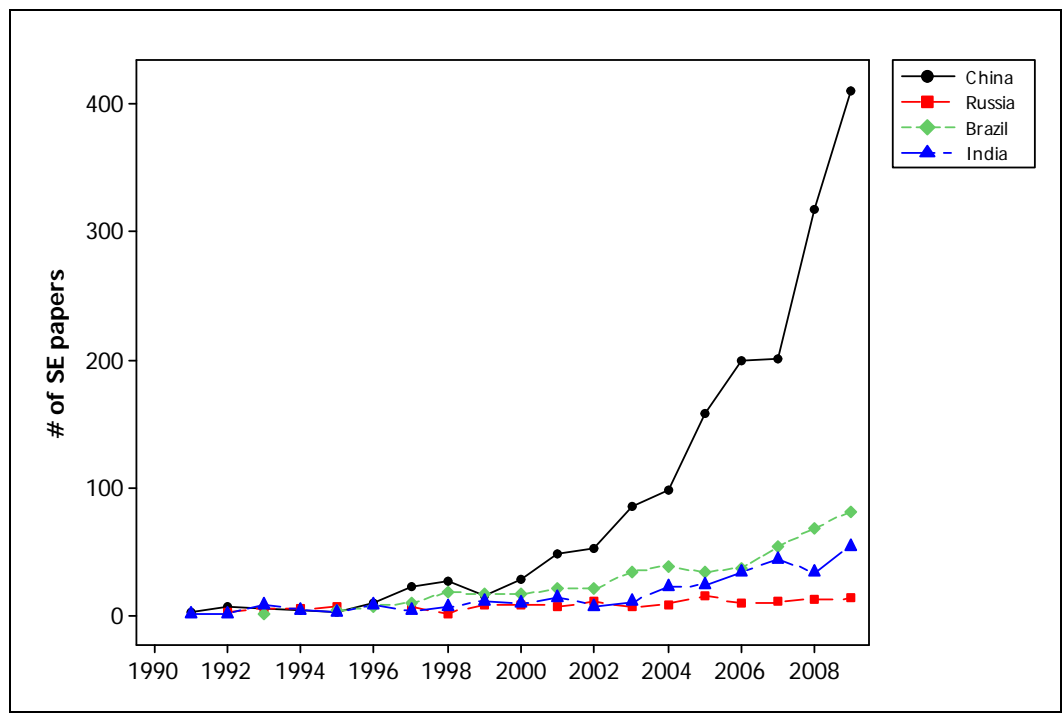

Fig. 14. SE research output of the BRIC countries (Brazil, Russia, India, and China).

\subsubsection{Middle Eastern Countries}

Most researchers in the SE area have the impression that there are not much SE research activities in the Middle East. We thus looked at this region's contributions and results are shown in Fig. 15. The main observations from this figure are discussed below.

- In the year 2009, about 80 (or just 3\%) of the total set of 2,092 indexed papers were from the Middle East, confirming that the Middle East's share of contributions is very small.

- Unlike most other countries in this region, Israel has had an active role in contributing papers from early 1990's. Perhaps, this is due to the high percentage of GDP spent in Israel on research and development, which was $4.46 \%$ as of 2004, the highest percentage among all countries in the world [36].

- A few countries such as Iran are sharply increasing their contributions in the recent years.

- Countries such as Yemen, UAE, Iraq or Bahrain have had no or very little contributions.

- Jordan has recently started to become an active contributor. 


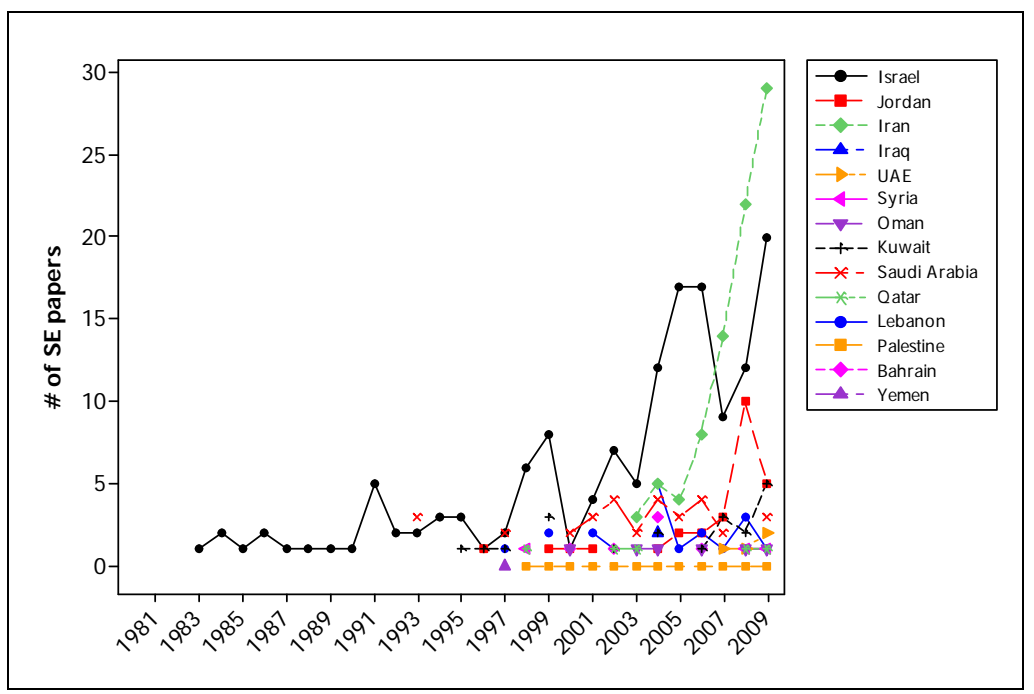

Fig. 15. SE research contributions of the Middle East countries.

\subsection{RQ 6: Comparing to Research Output in All Areas of Science}

It would be interesting to compare the trend of SE research output in each country with those of all other areas of science. For this purpose, the statistics related to all areas of science have been taken from the study reported in [16] which itself has extracted the information from the WoK [17]. Note that the notion of "science" here includes all the 256 disciplines covered by the WoK database which are the knowledge areas of in science, engineering, arts, and humanities [17].

As an example comparison, the trends of SE research output versus other scientific areas for 2000-2009 are depicted in Fig. 16. For brevity, we have only shown the top-5 countries (US, UK, China, Canada, and Germany). Note that these figures have dual yaxes and numbers of papers for the other areas of science are shown on the secondary yaxis (on the right-hand side).

The curves for the other scientific areas appear to be "smoother" than those for SE. The reason is that, statistically speaking, the number of papers in the other areas (in 200,000 's) is much higher than those for SE (less than 450 per year). Thus, one would expect that different factors (e.g., reduction in researching funding in a country) would have a "bumpier" impact on the SE curves, compared to the cumulative values for all the other areas.

Nevertheless, one can see similar trends between the two curves in each country. For example, the Chinese contributions in all scientific areas are on the rise lately, and that includes SE. However, the SE papers growth rate for China is faster than its trend of all scientific areas combined, which seems to denote that SE has been getting more attention in China more recently. For Canada and the US, no clear trend can be concluded on whether the SE papers' growth rate is higher or lower than all scientific areas combined. 


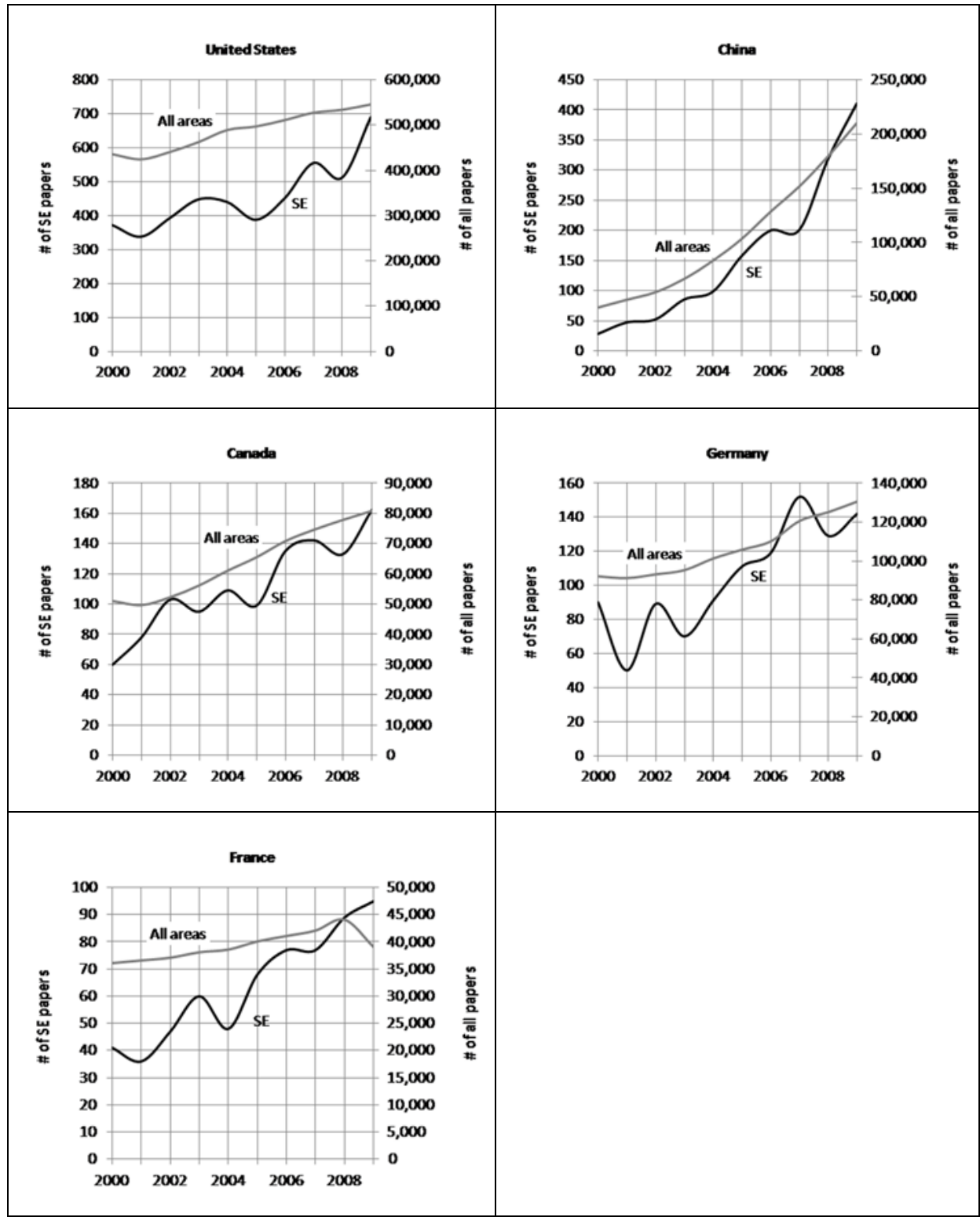

Fig. 16. Comparing SE research output to that of all other areas of science for top-5 countries, 2000-2009.

\section{Conclusion and Future Work}

Bibliometric studies are an established means to measure and analyze publications in an area of research. Because of the pervasive character and continuously growing importance of software, the study of Software Engineering (SE), as the discipline 
studying “... a systematic, disciplined, quantifiable approach to the development, operation, and maintenance of software" [39], is of substantial interest. While there is a growth of SE papers in general, this study looks more deeply intro temporal and regional trends within SE and in comparison to other disciplines. While there are other types of bibliometric studies in the SE community (e.g., [1-15]), no study to date has conducted the types of analysis we conducted in this article.

Based on the analysis of 26,624 papers, six research questions were studied in this article. The bibliometric study revealed interesting insights into the contributions of different countries and regions (e.g., China, and the EU countries) to the field of software engineering from 1969-2009. Among others, these insights are considered important for decisions about composition of program committees of conferences and editorial boards of journals. The results can also be used to see the impact and further need of regional funding initiatives.

Note that this geographic bibliometric study is considered an initial step in bibliometric analysis of the 40 years of software engineering research, and further investigations on the topic using further bibliometric techniques, metrics and concepts are necessary. The nature of this analysis is very much quantitative in terms of the number of publications. The qualitative impact in general is harder to measure, and is seen as a topic of future research in this area. We also plan to conduct other additional analyses, e.g., involvement of industry in publications by counting the ratio and number of practitioners being involved as authors of papers.

\section{Acknowledgments}

This project was supported by the Discovery Grant no. 341511-07 from the Natural Sciences and Engineering Research Council of Canada (NSERC) and also by the Alberta Ingenuity New Faculty Award no. 200600673. Vahid Garousi was further supported by the Visiting Scientist Fellowship Program (\#2221) of the Scientific and Technological Research Council of Turkey (TÜBİTAK).

\section{References}

[1] R. L. Glass and T. Y. Chen, "An Assessment of Systems and Software Engineering Scholars and Institutions (1996-2000)," Journal of Systems and Software, vol. 59, pp. 107-113, 2001.

[2] R. L. Glass and T. Y. Chen, "An Assessment of Systems and Software Engineering Scholars and Institutions (1997-2001) " Journal of Systems and Software, vol. 64, pp. 79-86, 2002.

[3] R. L. Glass and T. Y. Chen, "An Assessment of Systems and Software Engineering Scholars and Institutions (1998-2002) " Journal of Systems and Software, vol. 68, pp. 77-84, 2003.

[4] R. L. Glass and T. Y. Chen, "An Assessment of Systems and Software Engineering Scholars and Institutions (1999-2003) " Journal of Systems and Software, vol. 76, pp. 91-97, 2005.

[5] T. H. Tse, T. Y. Chen, and R. L. Glass, "An Assessment of Systems and Software Engineering Scholars and Institutions (2000-2004)," Journal of Systems and Software, vol. 79, pp. 816-819, 2006.

[6] W. E. Wong, T. H. Tse, R. L. Glass, V. R. Basili, and T. Y. Chen, "An Assessment of Systems and Software Engineering Scholars and Institutions (2001-2005)," Journal of Systems and Software, vol. 81, pp. 1059-1062, 2008. 
[7] W. E. Wong, T. H. Tse, R. L. Glass, V. R. Basili, and T. Y. Chen, "An Assessment of Systems and Software Engineering Scholars and Institutions (2002-2006)," Journal of Systems and Software, vol. 82, pp. 1370-1373, 2009.

[8] W. E. Wong, T. H. Tse, R. L. Glass, V. R. Basili, and T. Y. Chen, "An Assessment of Systems and Software Engineering Scholars and Institutions (2003-2007 and 2004-2008)," Journal of Systems and Software, vol. 84, pp. 162-168, 2011.

[9] J. Ren and R. N. Taylor, "Automatic and Versatile Publications Ranking for Research Institutions and Scholars," Communications of the ACM, vol. 50, pp. 81-85, 2007.

[10] V. Garousi and T. Varma, "Bibliometric Assessment of Canadian Software Engineering Scholars and Institutions (1996-2006)," Canadian Journal on Computer and Information Science, vol. 3, pp. 19-29, May 2010.

[11] K. W. McCain, J. M. Verner, G. W. Hislop, W. Evanco, and V. Cole, "The use of bibliometric and knowledge elicitation techniques to map a knowledge domain: Software Engineering in the 1990s," Springer Scientometrics, vol. 65, pp. 131-144, 2005.

[12] R. L. Glass, I. Vessey, and V. Ramesh, "Research in software engineering: an analysis of the literature," Information and Software Technology, vol. 44, pp. 491-506, 2002.

[13] K.-Y. Caia and D. Card, "An analysis of research topics in software engineering - 2006," Journal of Systems and Software, vol. 81, pp. 1051-1058, 2008.

[14] C. Wohlin, "An analysis of the most cited articles in software engineering journals - 2000," Information and Software Technology, vol. 49, pp. 2-11, 2007.

[15] C. Wohlin, "An analysis of the most cited articles in software engineering journals - 2001," Information and Software Technology, vol. 50, pp. 3-9, 2008.

[16] E. Archambault, "30 Years in Science: Secular Movements in Knowledge Creation," www.science-metrix.com/30years-Paper.pdf, Last accessed: April 2010.

[17] "ISI Web of Knowledge - Search Engine," http://apps.isiknowledge.com, Last accessed: April 2010.

[18] B. A. Galler, "ACM President's Letter: NATO and Software Engineering?," Communications of the ACM, vol. 12, p. 301, 1969.

[19] D. L. Parnas, "Stop the numbers game," Communications of the ACM, vol. 50, pp. 19-21, 2007.

[20] B. Meyer, C. Choppy, J. Staunstrup, and J. v. Leeuwen, "Viewpoint: research evaluation for computer science," Communications of the ACM, vol. 52, pp. 31-34, 2009.

[21] R. M. Geist, M. Chetuparambil, S. Hedetniemi, and A. J. Turner, "Computing research programs in the US," Communications of the ACM, vol. 39, pp. 96-99, 1996.

[22] J. Ren and R. N. Taylor, "A Java Tool for Ranking Institutions and Authors by Publications," www.isr.uci.edu/projects/ranking, Last accessed: Aug. 2009.

[23] Y. Jia and M. Harman, "An Analysis and Survey of the Development of Mutation Testing," IEEE Transactions on Software Engineering, To appear.

[24] M. Harman, S. Mansouri, and Y. Zhang, "Search based software engineering: A comprehensive analysis and review of trends techniques and applications," King's College London, Technical Report TR-09-03 http://www.dcs.kcl.ac.uk/technical-reports/papers/TR09-03.pdf, 2009.

[25] M. E. Falagas, E. I. Pitsouni, G. A. Malietzis, and G. Pappas, "Comparison of PubMed, Scopus, Web of Science, and Google Scholar: strengths and weaknesses" The FASEB Journal, vol. 22, pp. 338-342, 2008.

[26] É. Archambault, D. Campbell, Y. Gingras, and V. Larivière, "Comparing Bibliometric Statistics Obtained From the Web of Science and Scopus," Journal of American Society for Information Science, vol. 60, pp. 1320-1326, 2009.

[27] Y. Gu, "Global knowledge management research: A bibliometric analysis," Scientometrics, vol. 61, pp. 171-190, 2004. 
Xie,

"Software

Engineering

Conferences," http://people.engr.ncsu.edu/txie/seconferences.htm, Last accessed: Oct. 2011.

[29] I. H. Witten, E. Frank, and M. A. Hall, Data Mining: Practical Machine Learning Tools and Techniques: Elsevier, 2011.

[30] J. E. Hirsch, "An Index to Quantify an Individual's Scientific Research Output," Proceedings of the National Academy of Sciences, vol. 102, pp. 16569-16572, 2005.

[31] E. Garfield, "The Agony and the Ecstasy - The History and the Meaning of the Journal Impact Factor," presented at the International Congress on Peer Review in Biomedical Publication, 2005.

[32] World Bank, "Gross domestic product (2009)," The World Bank: World Development Indicators database, Retrieved 2010-07-05.

[33] B. Druss and S. Marcus, "Tracking publication outcomes of National Institutes of Health grants," American Journal of Medicine, vol. 118, pp. 658-663, 2005.

[34] M. Gaughan and B. Bozeman, "Using curriculum vitae to compare some impacts of NSF research grants with research center funding," Research Evaluation, vol. 11, pp. 17-26, 2002.

[35] V. Larivière, B. Macaluso, E. Archambault, and Y. Gingras, "Which scientific elites? On the concentration of research funds, publications and citations," Research Evaluation, vol. 19, pp. 45-53, 2010.

[36] World Bank database, "Research and development expenditure (\% of GDP)," http://data.worldbank.org/indicator/GB.XPD.RSDV.GD.ZS, Last accessed: Nov. 2012.

[37] S. Borodina and O. Shvyrkov, Investing in BRIC countries: Evaluating Risk and Governance in Brazil, Russia, India, and China: McGraw-Hill, 2010.

[38] D. Wilson and R. Purushothaman, "Dreaming with BRICs: The Path to 2050," Goldman Sachs Co., Report Number 99, http://www2.goldmansachs.com/ideas/brics/book/99dreaming.pdf, 2003.

[39] A. Abran, P. Bourque, R. Dupuis, and J. W. Moore, Guide to the Software Engineering Body of Knowledge (SWEBOK): IEEE Press, 2001.

[40] ACM, "ACM Computing Classification System (1998 Version, Valid through 2011)," http://www.acm.org/about/class/1998, Last accessed: Nov. 2011. 